\title{
A Comparative Study of Lithium Abundances, Radial Velocities, and Emission Lines in Pre-Main Sequence Visual Binaries
}

\author{
EDUARDO L. MARTÍN \& RAFAEL REBOLO \\ Instituto de Astrofísica de Canarias, E-38200, La Laguna, Spain \\ ANTONIO MAGAZZÙ \\ Osservatorio Astrofisico di Catania, I-95125 Catania, Italy
}

\begin{abstract}
High resolution ( $R \sim 20000$ ) spectroscopic observations of visual pairs of T Tauri stars (TTS) in the spectral range 655-675 nm, and 385-405 nm for some systems, are reported. Good seeing allows us to resolve pairs with minimum angular separation of $\sim 2^{\prime \prime}$. The radial velocities, overall spectral properties and detection of the $\mathrm{LiI}$ line are used to decide whether the components of the binaries are likely to be gravitationally bounded. In this paper we present first results on a subset of our sample: 3 visual companions are not TTS, namely DL Tau/c, GG Tau/c(SW) and NTTS $45251+3016 / c$. The star GG Tau/c(S) is confirmed as a T Tauri star. The system UZ Tau is probably composed of at least 4 components. The star 1 E0255.3+2018 (Fleming et al. 1989), previously thought to be single, is found to be a close visual binary. Finally, lithium abundances for the PMS components of 6 binaries are presented and their consistency with theoretical expectations is briefly discussed.
\end{abstract}

\section{INTRODUCTION}

Pre-main sequence (PMS) stars in binary systems are privileged observational sites for the study of star formation and early stellar evolution. They offer more constraints for comparing observations with theory because the components of physical pairs share many properties that are sources of uncertainty in single stars, e.g., reddening, metallicity, distance. Obviously it is necessary to check if the stars are actually physically associated. High resolution spectroscopy of each component is a powerful method as it allows to measure accurately radial velocities and line strengths. We use 3 criteria for classifying a visual binary as likely physical: small radial velocity difference $\left(\varsigma 10 \mathrm{~km} \mathrm{~s}^{-1}\right), \mathrm{H} \alpha$ in emission, and presence of the $\mathrm{LiI}$ resonance line at $670.8 \mathrm{~nm}$. We are considering only systems where the primary is a known TTS, because for Post T Tauri stars the situation may be different (Martín et al. 1992a). Lithium abundances of the components considered to be physical provide a check on the consistency of observations with theory. In a forthcoming paper we plan to place all the components in the $\mathrm{H}-\mathrm{R}$ diagram and compare them with evolutionary tracks and isochrones. We also intend to examine the mass-dependance of the level of activity and surface $\mathrm{Li}$ abundance, and to make a detailed comparison of our results with theoretical predictions of PMS lithium depletion. 


\section{RESULTS}

\subsection{Tau and NTTS $45251+3016$}

The secondaries of these 2 stars have $\mathrm{H} \alpha$ in absorption and the LiI line is not detected $\left(W_{\lambda} \leq 300 \mathrm{~m} \AA\right.$ and $W_{\lambda} \leq 40 \mathrm{~m} \AA$ for DL Tau/c and NTTS $45251 / \mathrm{c}$ respectively). Moreover they do not have molecular bands characteristic of M-type stars. They are classified according to the criteria given in the previous section as optical companions.

\subsection{GG Tau}

This star has 2 companions at $\sim 10^{\prime \prime}$ in the optical, one at $\mathrm{PA} 185^{\circ}$ and the other at PA $250^{\circ}$. We call them GG Tau/c(S) and GG Tau/c(SW) respectively. Only GG Tau/c(S) is seen in the NIR CCD frames of Moneti \& Zinnecker (1991). Our spectra confirm that GG Tau/c(S) is a T Tauri star with $\mathrm{H} \alpha$ in emission $\left(\mathrm{W}_{\lambda}=6.5 \mathrm{~nm}\right), \mathrm{HeI}$ at $\lambda 667.8 \mathrm{~nm}$ in emission $\left(\mathrm{W}_{\lambda}=0.2 \mathrm{~nm}\right)$, and the LiI doublet in absorption $\left(\mathrm{W}_{\lambda} \sim 50 \mathrm{pm}\right)$. The other companion GG Tau/c(SW) is a background hot star with $\mathrm{H} \alpha$ in absorption and $\mathrm{W}_{\lambda}(\mathrm{LiI}) \lesssim 25 \mathrm{pm}$.

\subsection{UZ Tau}

The two visual components of UZ Tau are well-known classical TTS (Cohen \& Kuhi 1979). They display many emission lines and the LiI line is fairly strong in both of them. However, our high resolution data show an intriguing radial velocity difference of $16 \pm 2 \mathrm{~km} \mathrm{~s}^{-1}$, which is the largest of all the PMS visual binaries we have examined so far. Looking at the literature we found that Edwards et al. (1987) had measured LiI rest velocities of $13.6 \mathrm{~km} \mathrm{~s}^{-1}$ for UZ Tau W and $1.2 \mathrm{~km} \mathrm{~s}^{-1}$ for UZ Tau E. We measured heliocentric radial velocities from the positions of several absorption lines including $\mathrm{LiI}$, and obtained $19 \mathrm{~km} \mathrm{~s}^{-1}$ for UZ Tau W and $35 \mathrm{~km} \mathrm{~s}^{-1}$ for UZ Tau E. Hence, the velocities for UZ Tau W are similar and close to the cloud radial velocity (Edwards et al.), but for UZ Tau E the discrepancy between our results and those of Edwards et al. are beyond the error bars. This star must be a spectroscopic binary. Recent lunar occultation observations (Simon et al. 1992) have shown that UZ Tau W is double. Therefore, the UZ Tau system seems to be a multiple system with at least 4 components. The complex nature of this system may help to explain some, if not all, of its extreme properties.

\subsection{A new T Tauri binary: 1 E0255.3+2018}

In the course of a survey of lithium in TTS (Martín et al. 1992b) we discovered in the TV camera of the $2.5 \mathrm{~m}$ Isaac Newton telescope that the object $1 \mathrm{E} 0255.3+2018$ is a close visual binary. Optical CCD frames were kindly taken upon request by R.M. González and E. Pérez at the $2.5 \mathrm{~m}$ Nordic Optical Telescope were the binary was clearly resolved. In our spectra both components show $\mathrm{H} \alpha$ in emission $\left(\mathrm{W}_{\lambda}=0.4 \mathrm{~nm}\right.$ and $0.06 \mathrm{~nm}$ ) and strong LiI absorption $\left(\mathrm{W}_{\lambda} \sim 50 \mathrm{pm}\right)$. Thus, we classify this system as a physical binary with weak $\mathrm{T}$ Tau components of similar luminosities. This object has the largest $\mathrm{X}$-ray flux and radius in the sample of Fleming et al. (1989). Its binary nature helps to account for its $\mathrm{X}$-ray properties. 


\subsection{Lithium abundances}

We present results on $\mathrm{Li}$ abundances for 5 out of the 6 visual naked TT binaries studied by Walter et al. (1988). Good seeing conditions allowed to resolve even the closest pair (NTTS $400012+2545$, Sep. $\approx 2^{\prime \prime}$ ). The Li abundances inferred from our measurements of $\mathrm{LiI}$ doublet equivalent widths are listed in the table below. The abundances take into account NLTE effects following calculations made by Dr. Pavlenko (private communication). We also present lithium abundances from observations of the binary V807-GH Tau.

\begin{tabular}{|c|c|c|c|c|}
\hline Star & $T_{e f f}(\mathrm{~K})$ & $\mathcal{M} / \mathcal{M}_{\odot}$ & $\operatorname{Age}\left(10^{6} y r\right)$ & $\log N(L i)$ \\
\hline $035120+3154 \mathrm{SW}$ & 5940 & 1.0 & $\approx 30$ & 3.4 \\
\hline $035120+3154 \mathrm{NE}$ & 5600 & 0.9 & $\approx 30$ & 3.2 \\
\hline $035135+2528 \mathrm{SW}$ & 4690 & 0.7 & $\approx 30$ & 2.6 \\
\hline $035135+2528 \mathrm{SE}$ & 4890 & 0.8 & $\approx 30$ & 3.0 \\
\hline $040012+2545 \mathrm{~N}$ & 4840 & 0.8 & $\approx \mathbf{3 0}$ & 3.5 \\
\hline $040012+2545 \mathrm{~S}$ & 4840 & 0.8 & $\approx \mathbf{3 0}$ & 3.3 \\
\hline $040047+2603 \mathrm{~W}$ & 3500 & 0.4 & 1.4 & $\leq 1.8$ \\
\hline $040047+2603 \mathrm{E}$ & 3500 & 0.4 & 1.6 & $<1.0$ \\
\hline $040142+2150 \mathrm{SW}$ & 3350 & 0.3 & 1.5 & $\leq 1.0$ \\
\hline $040142+2150 \mathrm{NE}$ & 3350 & 0.3 & 2.5 & $\leq 1.0$ \\
\hline V807 Tau & 4000 & 0.8 & 1.0 & 3.0 \\
\hline GH Tau & 3425 & 0.3 & $\leq 1.0$ & 2.8 \\
\hline
\end{tabular}

A detailed description of the methods for deriving stellar parameters and lithium abundances will be given in a forthcoming paper. The Li abundances of the TTS in visual pairs considered here have a spread of more than two orders of magnitude. Such a wide range is qualitatively consistent with theoretical expectations of Li depletion in PMS stars (Pinsonneault et al. 1990). Note that the internal differences in abundances between the components of each binary are small $(\leq 0.5$ dex.). Such differences are within the uncertainties of the derived abundances $( \pm 0.3 \mathrm{dex}$.$) . This is consistent with expectations for$ coeval components of similar masses.

\section{REFERENCES}

Cohen, M., Kuhi \& L.V. 1979, ApJS, 41, 743

Edwards, S, Cabrit, S., Strom, S.E., Ingeborg, H., Strom, K.M., \& Anderson, E. 1987, $A p J, 321,473$

Fleming, T.A., Gioia, I.M., \& Maccaro, T. 1989, ApJ, 340, 1011

Martín, E.L., Magazzù, A., \& Rebolo, R. 1992a, $A \mathcal{B} A, 257,186$

Martín, E.L., Magazzù, A., Rebolo, R., García López, R.J., \& Pavlenko, Ya.V. 1992b, in IAU Coll. No. 137, ed. W. Weiss

Moneti, A. \& Zinnecker, H. 1991, $A \& A, 242,428$

Pinsonneault, M.H, Kawaler, P., \& Demarque, P. 1990, ApJS, 74, 501

Simon, M., Chen, W.P., Howell, R.R., Benson, J.A., \& Slowik, D. 1992, ApJ, 384, 212

Walter, F.M., Brown, A., Mathieu, R.D., Myers, P.C., \& Vrba, F.J. 1988, $A J, 96,297$ 